\title{
Development of a reciprocating pump for space propulsion rocket system
}

\begin{abstract}
Space propulsion mainly uses reciprocating pumps that are fully integrated with the small scale applications. In this paper, the performance of a high-pressure pump as a feeding system for pressurizing fuel and an oxidizing to convey the flow and pressure in the combustion chamber will be evaluated. The use of this pump in jet engines is suitable for high environmental pressure and high ambient temperature as well as fuel injection pressure in various conditions. In this approach, the first step is to explain how the pump works; also we perform various modeling tests to check the performance of the pump. Numerical analysis of the stress and strain applied on the inner wall of the cylinder is performed according to its performance under internal pressure. It was seen that there is a smooth transition the stress is distributed along the cylinder wall thickness. The CFD modeling results were analyzed and the function that is expected from the pump was applied on the CAD model.
\end{abstract}

Volume 4 Issue 3 - 2020

Saboktakin A, Monjezi M, Qiassi S, Mehrjoi N

Department of Mechanical Engineering, University of Sistan and Baluchestan, Iran

\begin{abstract}
Correspondence: Saboktakin A, Department of Mechanical Engineering, University of Sistan and Baluchestan, Zahedan, Iran, Tel +989134348728, Email Saboktain@eng.usb.ac.ir
\end{abstract}

Received: July 25, 2020 | Published: August 14, 2020

\section{Introduction}

One of the important issues in flying objects is the high maneuverability, as well as the high efficiency to weight ratio. This feature can be improved by reducing the mass or increasing the specific impulse $\left(I_{\mathrm{sp}}\right)$ for engine. A low-mass fuel pump is being investigated, which can improve $\mathrm{I}_{\mathrm{sp}}$ by using high-pressure pumps while reducing propellant tank pressure. Furthermore, chemical advances are considered for missions that require high thrust to weight- ratio. If the consumption of the pump is low enough, it will not cause a propulsion failure, but it can be helpful to enhance the specific impulse of in-space propulsion systems. The purpose of the reciprocating pump is to reduce weight and operate at extremely high pressure and temperature, to reduce fuel consumption. As stated in table 1, another feature of the pump is to maintain full pressure in different conditions of space propulsion system.

Table I Applications and technical requirements of the reciprocating pump

\begin{tabular}{ll}
\hline Applications & $\begin{array}{l}\text { Small bipropellant or monopropellant rockets and developing an enabling technology for } \\
\text { Moon and Mars return missions }\end{array}$ \\
Pump type & Positive displacement pump driven by driver gas \\
Specifications & Generating a significant pressure rise while minimizing the loss from the driver gas
\end{tabular}

In 1990 Whitehead and his colleagues began research to build a high-pressure pump for use in research environments. Their goal was to build a light weight pump to supply fuel for spaceshipsthat designed to be shipped to Mars. First, in 1991 and 1993,,$^{1,2}$ they tested a sample of their design. The results were not promising. The pump was designed by the hydraulic signal valves, and displaced by the power of the piston shaft. Also, gas leakage in different conditions led to the decision to make changes to the control system and the shape of the design body of the pump, so they got better results. In 1995 Whitehead $^{3}$ evaluated its new pump and examined its results and performance. The results were relatively more promising than the original design of the pump. The weight and leakage of the pump was lighter and lower, respectively, instead of using hydraulic valves as well as pneumatic valve which made it easy for manufacturing and operating. At the end of $1997,{ }^{4}$ he analyzed the settings of this pump to climb Mars. Whitehead tested, evaluated, analyzed his results, between the years of 2001-2002 and 2007. .-9 $^{-9}$

In this paper, the first concentration will be on how the pump works, also, using ANSYS-FLUENT software, we perform various tests and check the performance of the pump. Finally, the results are analyzed and the function that is expected from the pump is checked.
The pump weight is around one percent of thrust. As stated above, the nature of first versions of reciprocating pump could not maintain full pressure without flow and had a little efficiency losses. Therefore, this research will be focused on the designing and developing a 300-gram pump could deliver fuel and oxidizer at $5 \mathrm{MPa}$ for $1000 \mathrm{~N}$ engine with remaining the tank pressure at $0.35 \mathrm{MPa}$.

\section{Pump design}

The pump has four cylinders and piston that joined together as shown in figure 1. It has a central section that deliver liquid inlet from the propellant tank to the pump. Directions indicate the inlet and outlet flows. The inlet port of the liquid propellant is the large port at the center of Figure 1, and the opposite side of the pump has separate outlet port. The gas is supplied to the outer cylinders and controlled by valves. As seen the figure 1, the liquid cylinders are lower in diameter than the gas cylinders. That area ratio enable to power its own reciprocating pump using delivered propellant.

At the end of each cylinder, there is a larger gas cylinder in diameter than liquid cylinder, and the entry point of the gas. Among them there is a piston separating fuel and gas chambers. As seen in the schematic of pump, it is known that there is no need for any shaft or 
other rotating part to apply gas pressure to the liquid. To cancel the mass influence of movement, the gas inlet valves are maintained, so that the opposite pistons movement are in toward or away each other.

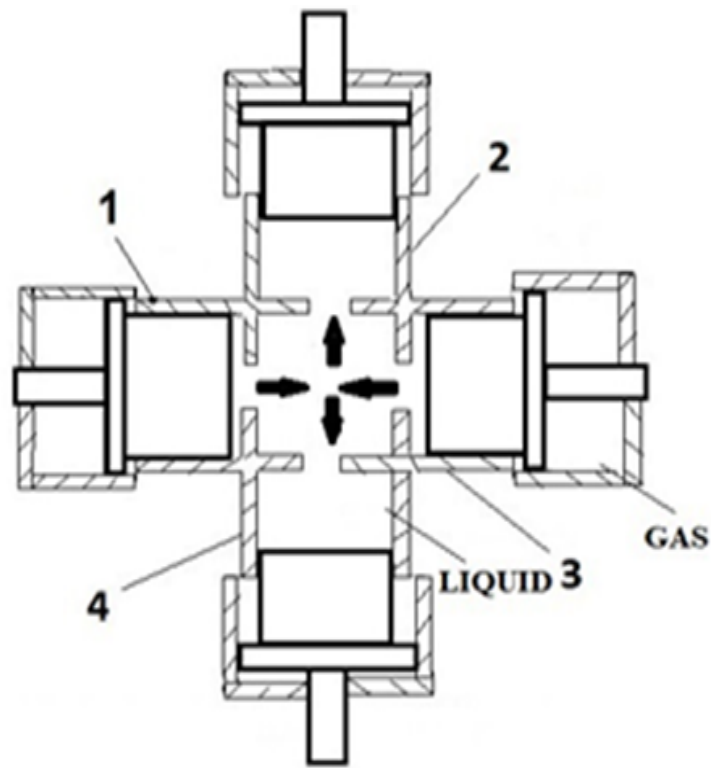

Figure I Primary schematics of the piston pump.

In addition, the control scheme is considered to compensate the pressure loss due to existing pistons in pump chambers. According to Figure 2, at first, cylinder numbers 1 and 3 are reaching at the end of the stroke, while the propellant has been refilled at cylinders 2 and 4 . The flow continuation is supplied by a control scheme so that cylinders 2 and 4 become pressurized with gas before pistons 1 and 3 are approaching their limit and their cylinders are vented.
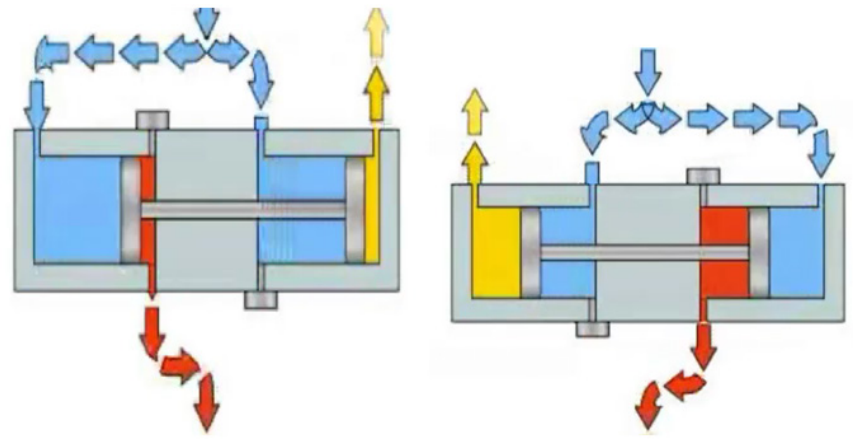

Figure 2 Arrangement of fuel injecting inside reciprocating pump from propellant tank to engine chamber.

The full sample of this pump weighs 470 grams. Blocks and cylinders, except for valves and tubes, are all made of aluminum. Large curved pipes have the task of sending gas to the inlet valves, which is used in the design of light alloy metals as shown in figure 3. The fuel entry site is the largest hole located at the center of the block and the exit point of the four small holes is placed in the center of the pump and on the other side. Each cylinder has an $8 \mathrm{cc}$ ideal displacement between the piston stops or $32 \mathrm{cc}$ per the cycle of the pump. Also, the valve opening time for fuel entry is less than $10 \mathrm{~ms}$, and this is independent of the speed of the piston. A key feature that was previously stated is that the diameter of the gas cylinder is larger than the liquid cylinder, to provide the necessary force to move the pistons. It should be noted that the gas and liquid cylinders are not integrated, but will be connected by screws as shown in figure 3 .
This pump operates at a much higher pressure than the one used in conventional launchers.

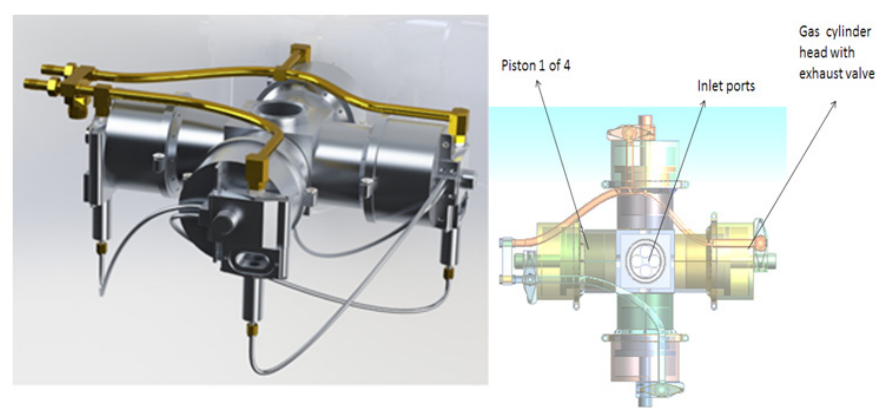

Figure 3 Initial design of the piston pump.

\section{Function of high pressure pump and energy conversion}

Figure 4 show an example of a propellant tanks which consists of a fuel tank and an oxidizing agent. Increasing the pressure by the pump is causing a very high propellant flow to the combustion chamber and delivers fuel and oxidizer at high pressure to produce high specific thrust. Each tank has a separate pump. The tank is designed to test at a length of $1.2 \mathrm{~m}$ and a wall thickness of $0.4 \mathrm{~mm}$. In fact, $25 \%$ of the mass of the launcher is made up of reservoirs. Another $25 \%$ is the engine, and $50 \%$ is specific to the fuel and oxidation of the propellant tanks. The pressure inside the propellant tank is about $0.35 \% \mathrm{MPa} .{ }^{6}$ In these tanks, there is the possibility of precise control of the mixture of two tanks including field and oxidizer can provide a lighter engine operating at a higher pressure, and more compact than a conventional engine having same thrust.

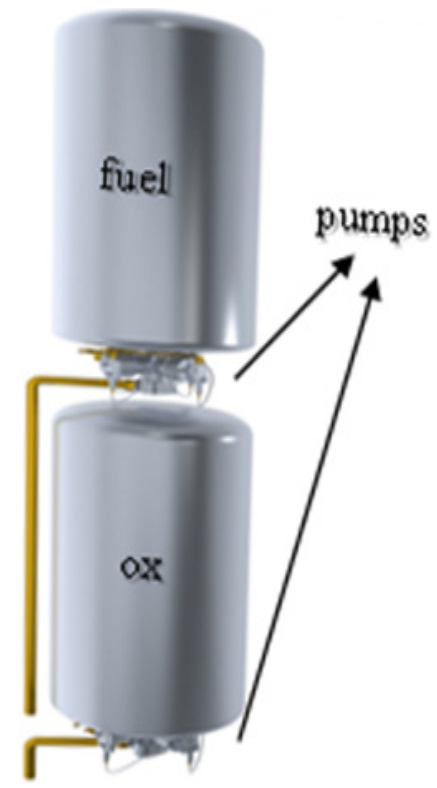

Figure 4 Low pressure tanks.

The pump performance is such that it receives a portion of the hydrazine fuel (about 2\%) and sends it to a gas generator with a one-way valve and a regulator at the initial start-up. As shown in figure 5, the gas generator is actually responsible for converting the hydrazine gas into gas based on the catalytic decomposition. After converting the energy of the gas at $4 \mathrm{Mpa}$, it forwarded to the pumps to instigate them. However, the fuel injection pressure on the engine will be between 5 and $6 \mathrm{MPa}$ when the pump is operating. As shown in Figure 5, there is no gas supply source, but gas is produced during 
the chemical process of the fuel itself used in the reservoirs, which will reduce the volume and mass of the launcher.

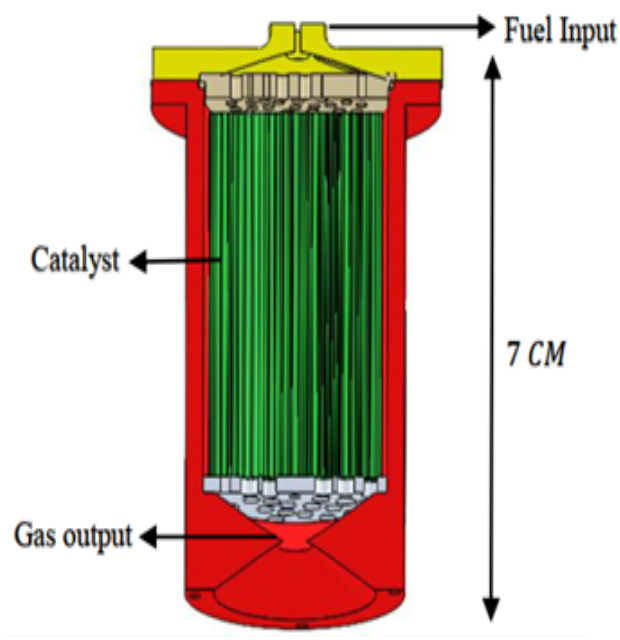

Figure 5 Hydrogen peroxide catalytic gas generator and its specifications.

\section{Difference pump with centrifuge pumps}

Conventional launchers mainly use pumps that include centrifugal pumps and turbines, which are fully integrated with the thrust chamber. A key requirement is that the pumps and their power supply should have a mass less than liquid inside the tank, engine, etc. Centrifugal pumps are shaft-driven by turbines and by scaling down these rotating pumps, they become less efficient and heavier. However, reciprocating pumps discussed in this paper are more suitable to small scale applications and those can provide wide ranges of flow and pressure.

As stated before, the goal is to design a light pump, in order to achieve a high acceleration in addition to achieving mass and volume. This is not possible with electric liquid propulsion engine is a motor where the chemical combustion is carried out using one or more liquid oxidizing and reducing safety factors. These regenerative compounds (fuels) and oxidizing factors are termed "propellant". This propellant is stored in tanks in the launcher, and when the rocket is turned on, the propellants are injected into the combustion chamber, causing combustion and thrust production. Another feature of this pump, which is not possible with turbo pumps, is that the system can maintain full pressure and fuel flow in any case. In other hand, in the case of electric pumps, the fuel is immediately disconnected when the power is interrupted. In fact, the pressure regulator along with the gas generator creates a guaranteed operation. These pumps are very simple and can work much more easily than small-scale turbopumps. In reciprocating pumps, the gas pressure is applied directly to the liquid, without the use of shafts or other components.

\section{Evaluation of results}

In this section we made engineering and research efforts with the help of advanced engineering commercial software (ANSYS, FLUENT, CATIA) to evaluate all parameters that affect the reciprocating pump design. These parameters are ported through a data management software linking, CFD and FEA. Simulation software's are directly connected to the CAD platform resulting in updates to the CAD model. This process allows an iterative design to develop a reliable pump design. It should be mentioned that due to the fact that the pump should be capable of functioning at a special ambient pressure and temperature even outside the Earth's atmosphere, we examined all these parameters and their influences using the Ansys CFX results.

The results including pressure distribution that conducted from the Ansys software have provided us with good answers, which are depicted in figures $6 \& 7$. These Figures indicate the pressure and velocity distribution at different locations in the flow from different ambient atmospheric pressure and gas temperature. These figures present the internal pressure and axial velocity profiles after initiation of propellant injection from tanks to combustion chamber. The peak pressure magnitude occurs at the larger piston surface and its walls. The peak velocity magnitude occurs at the middle of piston stroke in the cylinder.

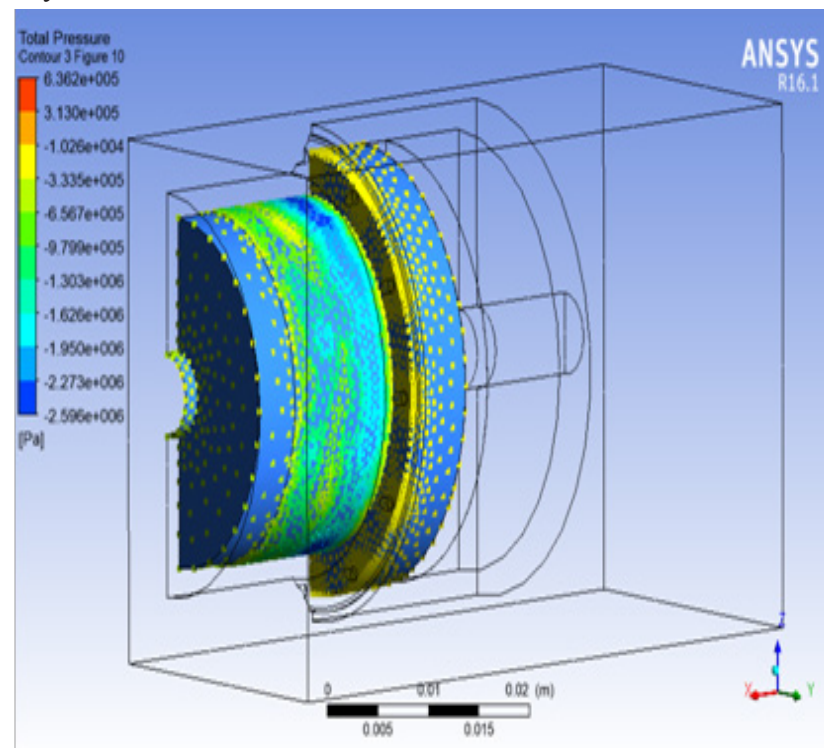

Figure 6 Pressure distribution along the piston surface.

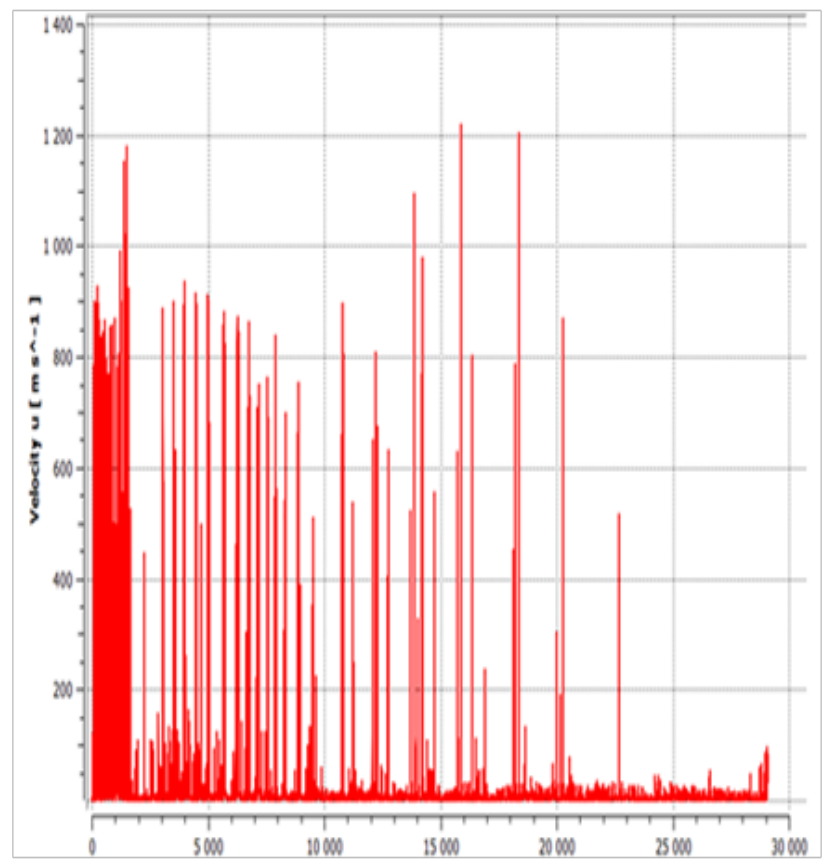

Figure 7 Velocity distribution profile along the piston stroke.

As seen the figure 8, we calculate the flow distribution in the flow around piston, distributed forces and resulting displacements. We adjust flow geometry around the piston to properly account for its 
spatial movement and evaluated changes flow distribution and flow forces/pressures.

The results for the stress and strain of the cylinder and piston according to the pressure exerted on the inner wall of the cylinder using finite element modeling are shown in figure 9. By taking the stress and strain outputs from a node and drawing the diagram for it, the strain diagram of the cylinder can be easily obtained.

According to results, it was concluded that although the pump looks simple, it's not possible to design and build at high speed. Possible errors include excessive mass, gas and fuel leakage from the vents, and leakage of the inlet and outlet valves. Also, in order to maintain the discharge pressure, it is necessary to reduce losses in the inlets and outlets of the liquid. Because, when a valve is opened, a significant amount of gas may be wasted. Proper mechanical levels should be selected for the desired lifetime and weight. Gaskets and the seat of the valves can be destroyed or deformed. The valves may bend. Liquid valves may be delayed and do not give us the desired returns.

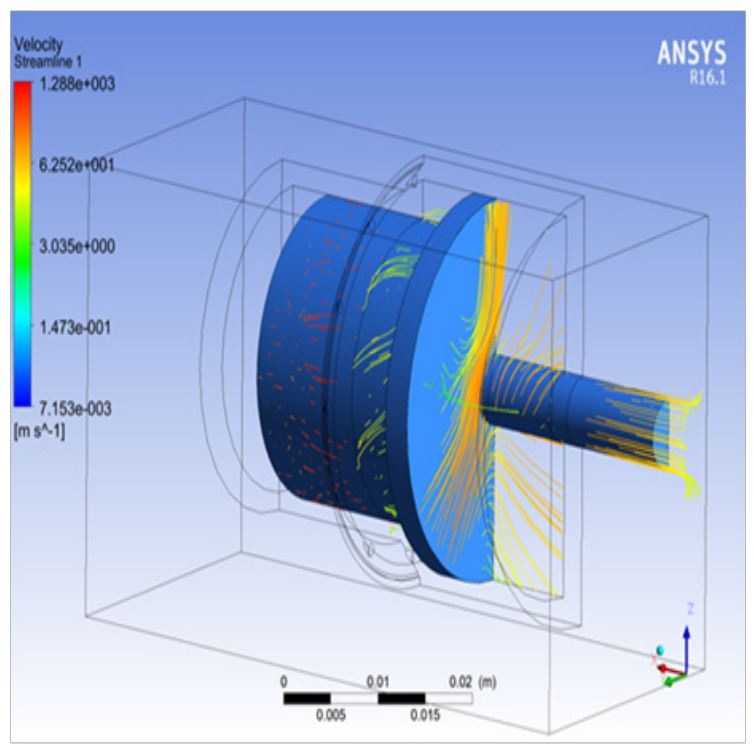

(a)

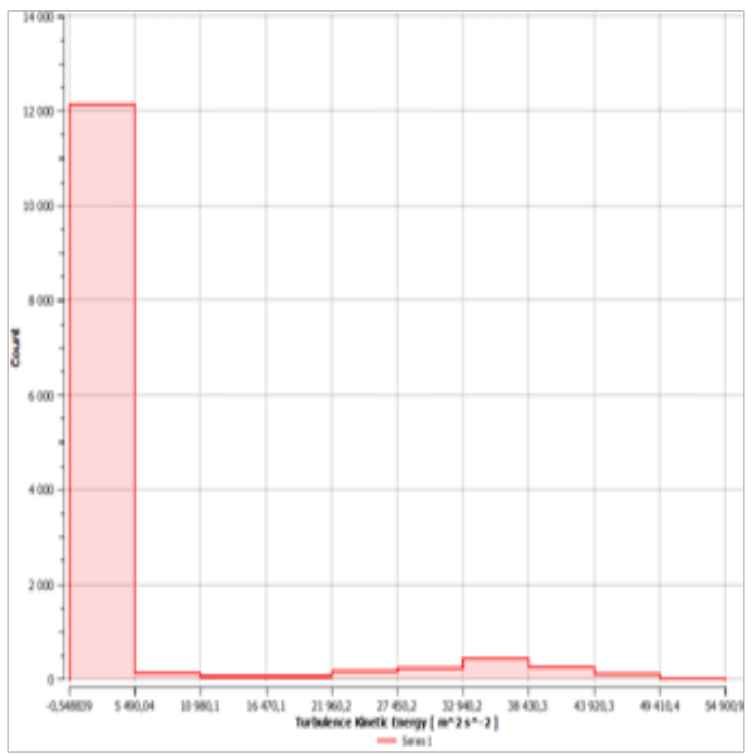

(b)

Figure 8 a) Gas flow distribution and b) Force distribution along the piston surface.

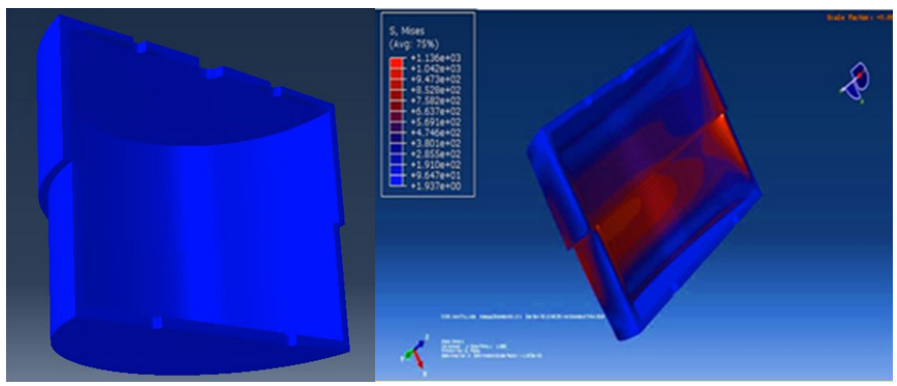

Figure 9 Stress distributions in half geometry of the inner wall of the cylinder showing maximum Von Mises stress.

\section{Conclusion}

Compared to other pumps, the reciprocated pump analyzed in this paper, has a lighter weight and a higher performance, which can have a positive impact on the acceleration and maneuverability of the launcher. According to a study, the precautions needed to build the pump should be avoided to prevent gas leakage into the fuel cylinder, because the hot gas has a high escape capability. The gas pipelines must be carefully designed and seamlessly integrated into the pipe as much as possible. Also, gas pipelines should be able to withstand extreme heat, which must be tested well before operation. We examined the internal environment of the pump, the piston, and the gas-to-piston collision rate with the Aansys-fluent software, and the results were almost satisfactory.

\section{Acknowledgments}

None.

\section{Conflicts of interest}

Authors declare that there is no conflict of interest.

\section{References}

1. Whitehead JC. United States Patent, Patent Number: 5,026,259, Date of Patent: Jun. 25, 1991.

2. Whitehead JC. United States Patent, Patent Number: 5,222,873, Date of Patent: Jun. 29, 1993.

3. Whitehead JC. Propulsion Engineering Study for Small-Scale Mars Missions. UCRL-CR-122442, Univ of Calif Lawrence Livermore National Lab; 1995.

4. Whitehead JC. Mars ascent propulsion options for small sample return vehicle. AIAA; 1997.

5. Whitehead JC. Hydrogen Peroxide Gas Generator Cycle with a Reciprocating Pump. AIAA Paper 2002-3702; 2002.

6. Gabermann M. Near Frictionless Air Cylinders Provide Precision Pneumatic Motion Control System. Power Conversion and Intelligent Motion. 1995;21(11):48-51.

7. Ventura M. Novel Concepts for an Advanced Non-Toxic Gas Generator. 42nd AIAA/ASME/SAE/ASEE Joint Propulsion Conference \& Exhibit Sacramento: CA; 2006

8. Lohner K, Dyer J, Doren E, et al. Design and Development of a SubScale Nitrous Oxide Monopropellant Gas Generator. AIAA 2007-5463, 43rd AIAA/ASME/SAE/ASEE Joint Propulsion Conference \& Exhibit: Cincinnati, $\mathrm{OH} ; 2007$.

9. Whitehead JC. Lightweight Quad Pump for Mars Ascent. Final Report to NASA Mars Technology Program for JPL contract no. 1263824, LLNL document no. 